Ann. Biol. anim. Bioch. Biophys., 1978, 18 (5), 1161-1172.

\title{
Evolution de l'adaptation métabolique des rats nourris avec des régimes hyperprotéiques
}

\author{
par M. CHANEZ, D. FAU, Brigitte BOIS-JOYEUX, J. PERET \\ Centre de Recherches sur la Nutrition du C.N.R.S., \\ 9, rue Jules-Hetzel, 92190 Meudon Bellevue.
}

Summary. Evolution of metabolic adaptation in rats fed high protein diets.

Three groups of 2-month old rats were fed for 40 days with diets containing 15, 50 or 75 p. 100 protein (casein). Seven rats in each group were killed after 1, 4, 8 and 40 days. Variations in plasma glucose and corticosterone, hepatic glycogen and liver enzyme activities of pyruvate kinase (PK), phosphoenolpyruvate carboxykinase (PEPCK), glucose-6phosphatase (G6Pase), alanine amino-transferase (GPT) and serine dehydratase (SDH) were studied. Food intake and body weight of rats fed high dietary protein levels were initially depressed but returned to normal after 4 to 8 days. Plasma glucose dropped at first, but returned rapidly to normal and remained steady until the end of the experiment. The level of hepatic glycogen also dropped 24 hrs after the dietary change, but rose after 4 to 8 days without reaching control levels. Plasma corticosterone concentration was greatly elevated for 1 day at the beginning of the high protein diets but decreased progressively back to normal within 8 days. During the first 8 days, the high protein level produced a progressive fall of PK activity ; this activity decreased in proportion to the dietary protein level. In the beginning of the adaptation period, G6Pase and PEPCK activities increased and rose simultaneously. As the animals gradually adapted to the high protein diet, the activity of these enzymes varied the opposite way; G6Pase returned to the initial level, while PEPCK stabilized at a high level proportional to that of the dietary protein. GPT and SDH activities increased progressively with protein intake, reaching a maximum at about 8 days. High activities were subsequently maintained until the end of the experiment.

\section{Introduction.}

De nombreux travaux montrent les effets des régimes riches en protéines sur l'activité des enzymes impliquées dans différentes voies métaboliques (Harper, 1965 ; Freedland et Szepesi, 1971). Cependant, dans la majorité des cas, les résultats sont obtenus à partir d'expériences fragmentaires, de courte durée, et dans des conditions expérimentales, souvent, peu comparables. Ces aspects nous ont incités à étudier par des expériences de longue durée les effets des variations du taux protéique de la ration sur les activités enzymatiques suivantes : pyruvate kinase (glycolyse), glucose-6phosphatase, phosphoénolpyruvate carboxykinase (néoglucogenèse), alanine-aminotransférase, sérine déshydratase (catabolisme protéique). En outre des mesures du glucose ef de la corficostérone plasmatiques, ainsi que du glycogène hépatique ont été effectuées. 


\section{Matériel et méthodes.}

Animaux et protocole expérimental. - Les expériences sont effectuées sur 91 rats mâles Wistar CF, âgés de 8 semaines (180-200 g) qui dès leur arrivée (automne) au laboratoire sont logés dans des cages individuelles et maintenus dans les conditions contrôlées de température $\left(22 \pm 2{ }^{\circ} \mathrm{C}\right)$ et d'éclairage (12/12, lumière $07: 00$ à $\left.19: 00\right)$. Après avoir reçu ad libitum pendant 10 jours un régime à 15 p. 100 de protéines, ils sont répartis en trois groupes nourris respectivement avec des régimes contenant 15, 50 ou 75 p. 100 de protéines. Dans chacun d'eux, des lots de 7 rats sont sacrifiés par décapitation entre 09 et 10 h) après 24 h, 4,8 ef 40 jours. Le sang est recueilli sur héparine et le plasma séparé par centrifugation est conservé à $-20^{\circ} \mathrm{C}$. Par ailleurs, les foies prélevés sont rapidement pesés, puis conservés à $-80^{\circ} \mathrm{C}$. Pendant la période expérimentale, la consommation de nourriture ef les variations pondérales sont mesurées.

Régimes. - Le régime à 15 p. 100 de protéines avait la composition suivante (en $\mathrm{g} / 100 \mathrm{~g}$ de régime) : caséine lactique (83,0 p. 100 de protéines) 19,9; amidon 65,1; huile d'arachide 8,0; mélange salin (Hubbel et al., 1937) 4,0; mélange vitaminique (Peret et al., 1973) 1,0 et cellulose en poudre 2,0. L'augmentation du contenu en protéines des régimes est réalisé aux dépens du matériel glucidique (amidon).

Méthodes.

Plasma. Le dosage du glucose est effectué au Technicon (réduction du ferrycyanure de potassium). La corticostérone est mesurée selon Guillemin ef al. (1958).

Foie. Le glycogène est extrait à chaud dans $\mathrm{KOH} 30$ p. $100(\mathrm{p} / \mathrm{v})$ puis précipité et lavé à froid avec de l'éthanol. Après centrifugation, le surnageant est écarté et le glycogène (culot) est hydrolysé avec $\mathrm{HCl} 1,5 \mathrm{~N}$. Le glucose libéré est mesuré par la méthode enzymatique de Huggett ef Nixon (1957).

Le dosage de la glucose-6-phosphatase (EC 3.1.3.9) (G6Pase) est effectué sur l'homogénat total selon Harper (1963). Les activités de la pyruvate kinase (EC 2.7.1.40) (PK) et de l'alanine-amino-transférase (EC 2.6.1.2) (GPT) sont déterminées respectivement par les méthodes de Weber et al. (1965b) et Segal et Matsuzawa (1970) sur un surnageant obtenu par centrifugation à $50000 \times \mathrm{g}$ d'un homogénat de foie dans le saccharose 0,25 M. L'activité de la sérine déshydratase ( $E C$ 4.2.1.13) (SDH) est mesurée sur un surnageant obłenu par centrifugation à $50000 \times \mathrm{g}$ d'un homogénat de foie dans un tampon phosphate de potassium $0,1 \mathrm{M}(\mathrm{pH} 7,2)$ selon la méthode de Fallon et al. (1966), en utilisant le milieu d'incubation proposé par Suda et Nakawa (1971). La libération du NAD+ est mesurée à $37^{\circ} \mathrm{C}$ et $340 \mathrm{~nm}$ à l'aide d'un spectrophotomètre Gilford $300 \mathrm{~T}$.

L'activité de la phosphoénolpyruvate carboxykinase (EC 4.1.1.32) (PEPCK) est mesurée selon la technique de Chang et Lane (1966), la fixation du ${ }^{14} \mathrm{C}$ étant mesurée à l'aide d'un compteur à scintillation Packard Tricarb.

La détermination de la concentration en protéines dans l'homogénat total et dans les surnageants est réalisée par la méthode de Kjeldhal.

Les activités enzymatiques sont exprimées en nanomoles de substrat converti à $37^{\circ} \mathrm{C}$ par minute et par $\mathrm{mg}$ de protéines.

L'analyse statistique des résultats est effectuée selon Snedecor et Cochran (1967). 


\section{Résultats.}

Consommation de nourriture et évolution pondérale (fig. 1). - Pendant les quatre premiers jours, les groupes 50 et 75 p. 100 de protéines diminuent leur consommation et accusent une perte de poids, ces effets étant plus prononcés chez les rats nourris avec le régime le plus riche en protéines $(P<0,05)$. Ces phénomènes sont précoces et plus marqués le premier jour d'expérience. Par la suite ces animaux semblent « s'adapler » aux conditions nutritionnelles ef leur comportement se rapproche de celui des rats nourris avec le régime à 15 p. 100 de protéines. Néanmoins, leur consommation continue à être basse ef l'écart entre le gain de poids des groupes 50 et 75 p. 100 de protéines et celui à 15 p. 100 de protéines se maintient. Il en résulte qu'en fin d'expérience (40 jours) les animaux témoins sont plus lourds que ceux nourris avec les régimes hyperprotéiques $(15$ p. $100>50$ p. $100>75$ p. 100) $(P<0,05)$.


FIG. 1. - Consommation de nourrifure et gain de poids des rats nourris pendant 40 jours avec différents taux de protéine dans le régime. $a=$ consommation quotidienne de nourriture $; b=$ consommation quotidienne de protéine ; $c=$ gain de poids quotidien. Chaque colonne représente la moyenne (7 animaux) \pm SEM. 
Glycogène hépatique, glucose et corticostérone plasmatiques (fig. 2). - Le taux de glycogène du foie varie inversement avec la teneur en protéines du régime. Si au début de l'expérience les animaux nourris avec les régimes hyperprotéiques accusent une baisse fort importante du glycogène hépatique, celui-ci augmente et atteint des valeurs maximales après 4 à 8 jours, pour se maintenir à ce niveau jusqu'à la fin de l'expérience.

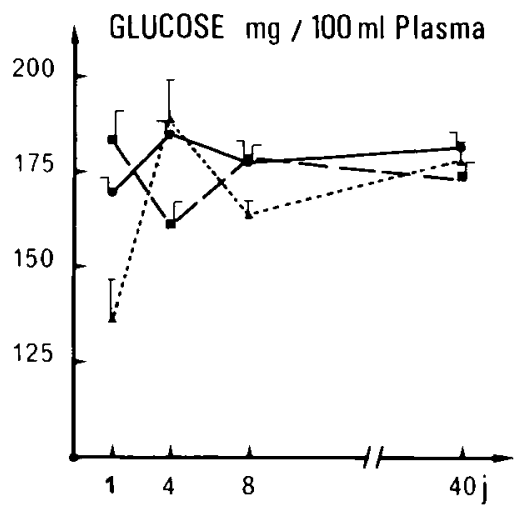

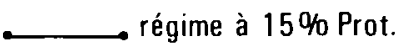

$-\rightarrow+. \quad 50 \%$

-................ . $75 \%$
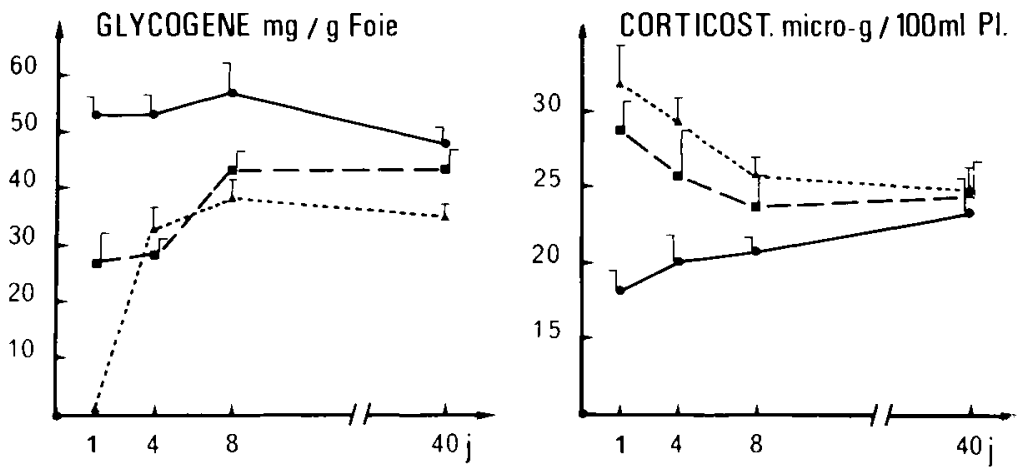

FIG. 2. - Variations du glycogène hépatique, du glucose ef de la corticostérone plasmatique de rats nourris pendant 40 jours avec différents faux de protéine dans le régime. Chaque point représente la moyenne (7 animaux) \pm SEM.

En dehors d'une baisse significative $(P<0,05)$ observée $24 \mathrm{~h}$ après le début de l'expérience chez les animaux nourris avec le régime à 75 p. 100 de protéines, le taux de glucose plasmatique se maintient relativement stable pendant toute la période expérimentale pour les trois groupes.

Dans les groupes 50 et 75 p. 100 de protéines, des valeurs élevées de corticostérone plasmatique s'observent $24 \mathrm{~h}$ après le début de l'expérience. Par la suite la corticostéronémie baisse, pour atteindre après 8 jours, le niveau de celle des rats nourris avec le régime à 15 p. 100 de protéines.

Activités enzymatiques hépatiques. - La figure 3 rend comple des activités des enzymes impliquées dans la glycolyse (PK), la néoglucogenèse (G6Pase, PEP-CK) et dans le catabolisme protéique (GPT, SDH). 
L'activité de la PK varie inversement avec le taux protéique du régime (fig. 3). La baisse de l'activité de l'enzyme est progressive, et d'autant plus importante que la consommation journalière de protéines est plus élevée. Dès que l'ingestion de nourriture des animaux consommant les régimes hyperprotéiques devient constante ( $8 \mathrm{e}$ jour), l'activité de la PK se stabilise jusqu'à la fin de l'expérience. Son activité représente alors seulement 43 et 23 p. 100 de celle des animaux témoins, respectivement pour les groupes nourris avec 50 et 75 p. 100 de protéines dans le régime.




FIG. 3. - Activités hépatiques de la pyruvate kinase, glucose-6-phosphatase, phosphoénolpyruvate carboxykinase, alanine amino transférase et sérine déshydratase de rats nourris pendant $\mathbf{4 0}$ jours avec différents taux de protéine dans le régime. Les activités enzymatiques sont exprimées en nanomoles de substrat converti à $37^{\circ} \mathrm{C}$ par minute et par $\mathrm{mg}$ de protéines. Chaque point représente la moyenne ( 7 animaux) \pm SEM. 
L'activité de la G6Pase augmente avec le taux protéique, mais retrouve son niveau initial avec l'écoulement du temps. Elle atfeint son niveau maximum dès le début de l'expérience $(24 \mathrm{~h})$. Pour les groupes nourris avec 50 et 75 p. 100 de protéines elle est augmentée respectivement de 45 et 85 p. 100. Par la suite, son activité diminue et s'approche dans les délais variant inversement avec le taux protéique de celle des animaux témoins : 4 jours chez les rats nourris avec le régime à 50 p. 100 de protéines ef 8 jours chez ceux recevant le régime à 75 p. 100 de protéines.

L'activité de la PEP-CK varie avec le taux protéique du régime, et atteint son maximum $24 \mathrm{~h}$ et 4 jours après le début de l'essai, respectivement pour les groupes nourris avec 50 et 75 p. 100 de protéines. Par la suite, lorsque la consommation des rats se stabilise (après 4 et 8 jours) l'activité de l'enzyme diminue $(P<0,05)$ tout en restant fort élevée par rapport à son niveau initial (augmentation de 1,9 et 2,5 fois). Ces niveaux d'activité restent constants jusqu'à la fin de l'expérience.

Les activités de la GPT et de la SDH vont de pair avec la consommation de protéines. Après une augmentation progressive fort importante jusqu'aux $4 \mathrm{e}$ et $8 \mathrm{e}$ jours d'expérience (stabilisation de la consommation), l'activité de la GPT continue à s'accroître faiblement, alors que celle de la SDH devient relativement stable jusqu'à la fin de l'expérience.

\section{Discussion.}

Les résultats concernant les effets du taux de protéines du régime sur la consommation de nourriture et l'évolution pondérale confirment les observations de la littérature (Harper, 1965). En fonction de la teneur en protéines du régime, après 4 à 8 jours, les animaux s'adaptent au régime en ce sens qu'ils retrouvent en grande partie le niveau initial de consommation (Mackay et al., 1941 ; Anderson ef al., 1968 ; Rogers ef Leung, 1973). Cependant, bien que de nombreux travaux aient étudié l'effet dépressif et transitoire des régimes hyperprotéiques sur la consommation de nourriture, les causes de ce phénomène sont loin d'être élucidées (Harper, 1976). Néanmoins, il faut souligner que le niveau de consommation a tendance à redevenir normal, lorsque les activités des enzymes impliquées dans la néoglucogenèse ef le catabolisme protéique, qui augmentent au cours de la période d'adaptation, ont elles aussi tendance à se stabiliser. Le parallélisme entre ces deux phénomènes est évident. Toutefois, dans l'état actuel de nos connaissances il est prématuré d'établir entre eux des relations de causalité. En outre, malgré la reprise de leur consommation, les animaux nourris avec les régimes hyperprotéiques n'arrivent pas à rattraper le gain pondéral des animaux de contrôle. Cela s'explique par l'incapacité de leur régime à assurer une couverture énergétique suffisante (Munro, 1964 ; Peret et al., 1975).

La baisse du glucose plasmatique ef l'absence de glycogène hépatique observées au début de l'expérience chez les rats nourris avec le régime à 75 p. 100 de protéines sont dues à la diminution brutale et fort importante de leur consommation. Cependant il faut souligner le rétablissement rapide de l'homéostasie glycémique (4 jours) malgré l'insuffisance de l'apport énergétique de leur régime. Par contre, celle-ci se reflète sur la teneur en glycogène du foie, qui même après l'adaptation des animaux (au régime), reste inférieure à celle des rats consommant le régime à 15 p. 100 de protéines. L'expli- 
cation de ces phénomènes doit être mise en relation avec les mécanismes de glycogénolyse et de glycogénosynthèse d'une part, et avec la mise en œuvre du processus de néoglucogenèse d'autre part.

Dans l'état actuel de nos connaissances la glycogénolyse ef la glycogénosynthèse sont en étroite relation avec le taux de glucose plasmatique, celui-ci étant le stimulus primitif déterminant la captation ou la libération du glucose du foie (Bergman et Bucolo, 1974). En outre, elles dépendent de la concentration en glucose ou en glucose6-phosphate (G6P) des cellules hépatiques, l'ensemble du système étant contrôlé par différentes hormones (insuline, glucagon, catécholamines, etc.) (Hers, 1976). Chez les rats nourris avec des régimes hyperprotéiques, au cours de la période d'adaptation, la consommation de nourriture est faible, le taux de glucose est bas, la glycogénolyse reste importante (surtout à $24 \mathrm{~h}$ ) et supplée en partie le manque de glucose. Cependant, les besoins en glucose sont couverts avant tout par la néoglucogenèse qui dès le début de la période d'adaptation est fort active. A partir du $4 \mathrm{e}$ jour, la production de G6P semble être suffisamment importante pour assurer le rétablissement de l'homéostasie glycémique et fournir du substrat pour la reconstitution du glycogène hépatique.

Lorsque les rats commencent à s'adapter, leur consommation de nourriture approche le niveau initial. Néanmoins, l'apport exogène de glucose reste soit faible (régime à 50 p. 100 de protéines) soit nul (régime à 75 p. 100 de protéines). L'homéostasie glycémique est maintenue par la néoglucogenèse, de même que la glycogénosynthèse. Cependant élant donné que le taux de glucose circulant est redevenu normal, la production de G6P se stabilise (Newsholme et Gevers, 1967) et le niveau de glycogène hépatique reste inférieur à celui des animaux de contrôle. Ces phénomènes persistent jusqu'à la fin de l'expérience chez les animaux nourris avec le régime le plus riche en protéines. Chez les rats consommant le régime à 50 p. 100 de protéines il est probable qu'une partie du glucose ingéré est utilisé pour la synthèse du glycogène du foie, si bien qu'en fin d'expérience sa teneur approche celle des animaux témoins. L'ensemble de ces explications doit être confirmé par des mesures conséquentes concer nant les variations hépatiques des activités de la glycogène phosphorylase ef synthétase de même que du glucose-6-phosphate.

L'augmentation initiale brutale de la corticostérone plasmatique observée chez les rats nourris avec les régimes hyperprotéiques témoigne de l'intensification de l'activité des surrénales. Cette observation constitue une preuve directe de l'hypothèse déjà formulée par différents auteurs en se basant seulement sur l'augmentation du volume du cortex surrénalien (Leathem, 1964 ; Munro, 1964). Touł porte à croire que ce phénomène est lié au processus d'adaptation, puisque la corticostéronémie s'abaisse à mesure que les animaux s'adaptent, pour atteindre dès le $8 \mathrm{e}$ jour le niveau de celle des rats nourris avec le régime à 15 p. 100 de protéines. Par ailleurs, une évolution semblable de la corticostérone plasmatique a été observée lors de l'administration des régimes contenant un excès en méthionine (Munro et al., 1963, 1965 ; Peretianu, 1968 ; Girard-Globa et al., 1972).

Chez les rats nourris avec des régimes hyperprotéiques, la baisse progressive de l'activité de la PK au cours de l'adaptation est semblable à celle observée par d'autres auteurs (Szepesi et Freedland, 1967, 1968a, 1968b). Ces variations peuvent s'expliquer par les mécanismes de régulation de la PK, de la glycolyse et de la néoglucogenèse. Chez les animaux ayant une consommation élevée de glucose, la synthèse de l'enzyme 
est stimulée (Szepesi et Freedland, 1968b) et le phosphoénolpyruvate (PEP) substrat de la PK est obtenu par la glycolyse. Lorsque les animaux consomment des régimes riches en protéines, l'apport en glucose est faible, la glycolyse manque de substrat ef le PEP est obtenu à partir du pyruvate ou de l'oxaloacétate par la mise en route des processus de transamination et de néoglucogenèse. Cependant, au début de la période d'adaptation la voie de la glycolyse étant prédominante (Weber ef al., 1967 ; Weber, 1969) le PEP formé est recyclé en pyruvate sous l'action de la PK (Hers, 1976). Au fur et à mesure que les animaux s'adaptent aux conditions nutritionnelles, leur consom mation de nourriture augmente, de même que la production de pyruvate (et d'oxaloacétate) ; la néoglucogenèse prend alors le pas sur la glycolyse, le PEP produit est utilisé surtout pour la synthèse du glucose (PEP $\rightarrow 2$ phosphoglycérate $\rightarrow$ G6P $\rightarrow$ glucose) et l'activité de la PK est progressivement inhibée (Weber, 1969 ; Hers, 1976). Lorsque la consommation de nourriture approche le niveau initial, ces phénomènes se stabilisent; l'activité de la PK devient constante et est d'autant plus basse que le régime est plus riche en protéines (Krebs et Eggleston, 1965 ; Freedland ef al., 1966 ; Peret et al., 1975).

Les variations de l'activité de la G6Pase, GPT et SDH observées chez les rats nourris avec les régimes hyperprotéiques sont semblables à celles de la litférature (Freeland et Harper, 1957, 1958 ; Szepesi et Freedland, 1967, 1968a; Rosen et al., 1959, 1973 ; Fallon ef al., 1966 ; Freedland ef al., 1966 ; Anderson et al., 1968 ; Mauron ef al., 1973). L'augmentation de l'activité de la PEP-CK avec la teneur en protéines chez les animaux adaptés est un phénomène connu (Hanson et Garber, 1972) mais les variations de l'activité de l'enzyme au cours de l'adaptation n'ont pas été étudiées.

Bien que la G6Pase et la PEP-CK fassent partie de la néoglucogenèse, leurs activités augmentent rapidement et vont de pair seulement au début de la période d'adaptation. Par la suite, à mesure que les animaux s'adaptent aux conditions nutritionnelles leurs activités varient en sens inverse : la G6Pase retrouve son niveau initial alors que la PEP-CK se stabilise à un niveau élevé, proportionnel à la teneur en protéines du régime. Ces observations suggèrent deux mécanismes de régulation de l'activité des deux enzymes : (a) un mécanisme de stimulation, à courte durée, qui joue lors du changement brutal de régime et $(b)$ un mécanisme de maintien ou de répression, à long terme, qui se manifeste lors de l'adaptation des animaux au régime. Le mécanisme de stimulation implique, entre autres facteurs, une régulation hormonale, la corticostérone et le glucagon jouant probablement dans nos conditions expérimentales le rôle principal (Gelehrter, 1973). II est bien connu que l'administration des glucocorticoïdes entraîne après un laps de temps variable (minimum 4 à 6 h) l'augmentation de l'activité de la G6Pase (Weber et al., 1955, 1965a; Kvam et Parks, 1960 ; Freedland et Harper, 1966) ef de la PEP-CK (Shrago et al., 1963 ; Foster et al., 1966 ; Wicks ef al., 1969). Nos résultats semblent confirmer ces observations, puisque $24 \mathrm{~h}$ après le début des expériences les animaux nourris avec les régimes hyperprotéiques ont le taux le plus élevé de corticostérone plasmatique. Néanmoins, l'hormone surrénalienne ne paraît avoir qu'un rôle permissif, puisque l'augmentation de l'activité des deux enzymes s'observe aussi chez les rats surrénalectomisés (Freedland et Barnes, 1963 ; Shrago et al., 1963). Les variations de la glucagonémie lors de la consommation des régimes hyperprotéiques ont été peu étudiées (Eisenstein et Strack, 1976). Cependant, tout porte à croire que le glucagon joue un rôle essentiel dans le mécanisme de stimulation des deux enzymes. En effet, leurs activités sont augmentées au cours du jeû ne ou du diabète 
(Langdon et Weakley, 1955 ; Harper, 1959 ; Fitch et Chaikoff, 1961 ; Foster ef al., 1966), conditions qui s'accompagnent de taux élevés de glucagon (Unger ef Lefebvre, 1972) et de concentrations hépatiques élevées d'AMP cyclique (Jefferson et al., 1968; Park et Exton, 1972). Par ailleurs, l'administration du glucagon ou du « dibutyryl cyclic AMP " entraîne une augmentation de la PEP-CK (Shrago ef al., 1963 ; Wicks et al., 1969) celle-ci étant due à une synthèse accrue de l'enzyme (Wicks ef al., 1972 ; Hanson et al., 1973).

Le mécanisme de maintien à un niveau élevé de l'activité de la PEP-CK, lorsque les animaux sont adaptés aux régimes hyperprotéiques est relativement mal connu. Nos observations suggèrent que la corticostérone n'est pas impliquée dans ce processus, puisque la corticostéronémie élevée au début du changement de régime revient à des taux comparables à ceux des témoins lorsque les animaux sont adaptés au régime. Le rôle éventuel du glucagon reste à préciser, bien que chez des rats adaptés à des régimes riches en protéines la glucagonémie est légèrement supérieure à celle des animaux de contrôle (Eisenstein et al., 1974). Tout porte à croire, que chez les animaux adaptés, le contrôle de l'activité de l'enzyme s'exerce surtout par les substrats (pyruvate, oxaloacétate) provenant du catabolisme des acides aminés (Freedland et Szepesi, 1971 puisque la stabilisation de l'activité de la PEP-CK coïncide avec celle de la GPT et de la $\mathrm{SDH}$. Ce contrôle semble s'effectuer au niveau de la traduction, le rôle des substrats étant de stabiliser le mARN, induit au cours de l'adaptation (Kenney, 1970).

La baisse de l'activité de la G6Pase lors de l'adaptation des rats aux régimes hyperprotéiques s'explique par le fait que cette enzyme participe étroitement au contrôle de la glycémie (Newsholme et Gevers, 1967 ; Hers, 1976). Parmi d'autres facteurs, l'enzyme est stimulée par le G6P et réprimée par le glucose. Lorsque la glycémie est rétablie, l'activité de l'enzyme retrouve son niveau initial ef le G6P produit par la néoglucogenèse est utilisé pour la synthèse du glycogène (Hers, 1976).

Bien que lors de l'ingestion des régimes riches en protéines, la période d'induction des activités de la GPT ef de la SDH soit nettement plus longue que celle de la PEP-CK, la plupart des auteurs considèrent que les mécanismes de régulation de ces enzymes sont relativement semblables (Kenney, 1970 ; Freedland et Szepesi, 1971). Actuellement on pense que les différences de temps de réponse au changement de régime ou à l'administration d'hormones (glycocorticoïdes ou glucagon) reflètent les différents taux de renouvellement de ces enzymes (Schimke, 1970, 1975). La demi-vie de la PEP-CK est de l'ordre de 5 à 6 h (Treadow et Khairallah, 1972 c Hopgood ef al., 1973) alors que celles de la GPT et de la SDH sont plus longues, étant respectivement évaluées de $35 \mathrm{~h}$ à 3 jours (Kim, 1969 c Szepesi et Freedland, 1969) et de 20 à $30 \mathrm{~h}$ (Jost ef al., 1969 Szepesi et Freedland, 1969).

Nos observations confirment donc ces aspects théoriques puisque le niveau maximum d'activité de la PEP-CK est atteint plus rapidement ( $24 \mathrm{~h}$ à 4 jours) que celui de la GPT et de la SDH (4 à 8 jours).

En conclusion, les résultats obtenus dans des expériences de longue durée, chez des rats adultes nourris avec des régimes hyperprotéiques montrentl'existence de deux périodes distinctes :

a) La première dite "d'adaptation », dont la durée (4 à 8 jours) dépend de la teneur en protéines du régime, se caractérise par une diminution de la consommation et une perte de poids. En outre, on observe au lendemain du changement du régime 
une diminution du taux de glucose plasmatique et du glycogène hépatique et une élévation importante de la corticostéronémie. Par ailleurs, ces phénomènes s'accompagnent d'une baisse progressive de l'activité de la PK, d'une augmentation importante des activités de la G6Pase et de la PEP-CK et d'une augmentation progressive et fort importante de l'activité de la GPT et de la SDH.

b) La seconde période se caractérise par un retour à la normale de la consommation et du gain de poids. En outre, les taux de glucose et de corticostérone plasma tiques retrouvent leurs valeurs initiales, tandis que le taux de glycogène hépatique bien qu'il remonte, n'atteint pas les valeurs des animaux témoins. Par ailleurs, alors que l'activité de la PK se maintient à un niveau faible, celles de la GPT et de la SDH se stabilise à un niveau fort élevé. Enfin bien que la G6Pase et la PEP-CK fassent partie de la néoglucogenèse, leurs activités varient dans le sens inverse : la G6Pase retrouve son niveau initial, alors que la PEP-CK se stabilise à un niveau élevé proportionnel à la teneur en protéines du régime.

Reçu en janvier 1978. Accepté en mars 1978.

\section{Références}

ANDERSON H. L., BENEVENGA N. J., HARPER A. E., 1968. Associations among food and protein intake, serine dehydratase, and plasma amino-acids. Amer. J. Physiol., 214, 1008-1013.

BERGMAN R. N., BUCOLO R. J., 1974. Interaction of insulin and glucose in the control of hepatic glucose balance. Amer. J. Physiol., 227, 1314-1322.

CHANG H. C., LANE M. D., 1966. The enzymatic carboxylation of phosphoenol pyruvate. J. biol. Chem., 241, 2413-2420.

EISENSTEIN A. B., STRACK I., 1976. A nonsuppressible increase of glucagon secretion by isolated islets of high-protein-fed rats. Diabetes, 25, 51-55.

EISENSTEIN A. B., STRACK I., STEINER A., 1974. Glucagon stimulation of hepatic gluconeogenesis in rats fed a high profein, carbohydrate free diet. Metabolism, 23, 15-23.

FALLON H. J., HACKNEY E. J., BYRNE W. L., 1966. Serine biosynthesis in rat liver. Regulation of enzyme concentration by dietary factors. J. biol. Chem., 241, 4157-4167.

FITCH W. M., CHAIKOFF I. L., 1961. Effect of previous diet on response of hepatic enzyme activities to a 24-hour fast. Biochemistry, 94, 380-386.

FOSTER D. O., RAY P. D., LARDY H. A., 1966. Studies on the mechanisms underlying adaptive changes in rat liver phosphoenolpyruvate carboxykinase. Biochemistry, 5, 555-562.

FREEDLAND R. A., BARNES J. K., 1963. The effect of adrenalectomy on the adaptation of glucose-6phosphate metabolizing enzymes in liver. J. biol. Chem., 238, 1915-1918.

FREEDLAND R. A., HARPER A. E., 1957. Metabolic adaptation in higher animals. I. Dietary effects on liver glucose-6-phosphatase. J. biol. Chem., 228, 743-751.

FREEDLAND R. A., HARPER A. E., 1958. Metabolic adaptation in higher animals. Il. Changes with time in the adaptive response of glucose-6-phosphatase. J. biol. Chem., 230, 833-841.

FREEDLAND R. A., HARPER A. E., 1966. Initiation of glucose-6-phosphatase adaptation in the rat. J. Nutrit., 89, 429-434.

FREEDLAND R. A., SZEPESI B., 1971. Control of enzyme activity : nutritional factors, 351-358. In RECHCIGL M. Jr., Enzyme synthesis and degradation in mammalian systems. Karger, Basel.

FREEDLAND R. A., CUNLIFFE T. L., ZINKL J. G., 1966. The effect of insulin on enzyme adaptations to diets and hormones. J. biol. Chem., 241, 5448-5451.

GELEHRTER T. D., 1973. Mechanisms of hormonal induction of enzymes. Metabolism, 22, 85-100.

GIRARD-GLOBA A., ROBIN P., FORESTIER M., 1972. Long-term adaptation of weanling rats to high dietary levels of methionine and serine. J. Nutrit., 102, 209-218.

GUILLEMIN R., CLAYTON G. W., SMITH J. D., LIPSCOMB H. S., 1958. Measurement of free corticosteroids in rat plasma physiological validation of a method. Endocrinology, 63, 349-358. 
HANSON R. W., GARBER A. J., 1972. Phosphoenolpyruvate carboxykinase. I. Its role in gluconeogenesis. Am. J. clin. Nutrit., 25, 1010-1021.

HANSON R. W., FISHER L. M., BALLARD F. J., RESHEF L., 1973. The regulation of phosphoenolpyruvate carboxykinase in fetal rat liver. Enzyme, 15, 97-110.

HARPER A. E., 1959. Hormonal factors affecting glucose-6-phosphatase activity. 2. Some effects of diet and of alloxan diabetes in the rat. Biochem. J., 71, 702-705.

HARPER A. E., 1963. Determination of glucose-6-phosphatase, 788-792. In BERGMEYER H. V., Method of enzymatic analysis. Acad. Press, New York, London.

HARPER A. E., 1965. Effect of variation in protein intake on enzymes of amino acids metabolism. Canad. J. Biochem., 43, 1589-1608.

HARPER A. E., 1976. Protein and amino acids on the regulation of food intake, 103-113. In NOVIN D., WYRWICKA W., BRAY G., Hunger : basic mechanism and clinical implications, Raven Press, New York.

HERS H. G.,1976. The control of glycogen metabolism in the liver. Annu. Rev. Biochem., 45, 167-189.

HOPGOOD M. F., BALLARD F. J., RESHEF L., HANSON R. W., 1973. Synthesis and degradation of phosphoenolpyruvate carboxylase in rat liver and adipose tissue. Biochem. J., 134, 445-453.

HUBBEL R. B., MENDEL L. B., WAKEMAN A. J., 1937. A new salt mixture for use in experimental diets. J. Nutrit., 14, 273-275.

HUGGETT A. St. G., NIXON D. A., 1957. Enzymic determination of blood glucose. Biochem. J., 66, $12 \mathrm{p}$.

JEFFERSON L. S., EXTON J. H., BUTCHER R. W., SUTHERLAND E. W., PARK C. R., $1968 . \quad$ Role of adenosine $3^{\prime}, 5^{\prime}$-monophosphate in the effects of insulin and anti-insulin serine on liver metabolism. J. biol. Chem., 243, 1031-1038.

JOST J. P., KHAIRALLAH E. A., PITOT H. C., 1968. Studies on the induction and repression of enzymes in rat liver. J. biol. Chem., 243, 3057-3066.

KENNEY F. T., 1970. Hormonal regulation of synthesis of liver enzymes, 131-176. In MUNRO H. N., Mammalian protein metabolism, vol. 4. Acad. Press, New York, London.

KIM Y. S., 1969. Half-life of alanine amino transferase and of total soluble protein in livers of normal and glucocorticoid treated rats. Mol. Pharmacol., 5, 105-108.

KREBS H. A., EGGLESTON L. V., 1965. The role of pyruvate kinase in the regulation of gluconeogenesis. Biochem. J., 94, 3c-4c.

KVAM D. C., PARKS R. E. Jr., 1960. Hydrocortisone induced changes in hepatic glucose-6-phosphatase and fructose diphosphatase activities. Amer. J. Physiol., 198, 21-24.

LANGDON R. G., WEAKLEY D. R., 1955. The influence of hormonal factors and of diet upon hepatic glucose-6-phosphatase activity. J. biol. Chem., 214, 167-174.

LEATHEM J. H., 1964. Some aspects of hormone and protein metabolic interrelationships, 343-380. In MUNRO H. N., ALLISON J. B., Mammalian profein metabolism., vol. 1. Acad. Press, New York.

MACKAY E. M., BARNES R. H., CARNE H. O., 1941. The influence of a diet with a high proteine content upon the appetite and deposition of fat. Amer. J. Physiol., 135, 193-201.

MAURON J., MOTTU F., SPOHR G., 1973. Reciprocal induction and repression of serine dehydratase and phosphoglycerate dehydrogenase by proteins and dietary essential amino acids in rat liver. Eur. J. Biochem., 32, 331-342.

MUNRO H. N., 1964. General aspects of the regulation of protein metabolism by diet and by hormones, 385-481. In MUNRO H. N., ALLISON J. B., Mammalian protein metabolism. Acad. Press, New York.

MUNRO H. N., STEELE M. H., HUTCHISON W. C., 1963. Blood corticosterone levels in the rat after administration of amino acids. Nature (London), 199, 1182-1183.

MUNRO H. N., STEELE M. H., HUTCHISON W. C., 1965 . Action of dietary protein and amino acids on the rat adrenal gland. Brit. J. Nutrif., 19, 137-147.

NEWSHOLME E. A., GEVERS W., 1967. Control of glycolysis and gluconeogenesis in liver and kidney cortex. Vifam. Horm., 25, 1-86.

PARK C. R., EXTON J. H., 1972. Glucagon and the metabolism of glucose, 77-108. In : LEFEBVRE P. J. UNGER R. H., Glucagon, molecular physiology, clinical and therapeutic implications. Pergamon Press, New York.

PERET J., MACAIRE I., CHANEZ M., 1973. Schedule of protein ingestion, nitrogen and energy utiliza- 
tion and circadian rhythm of hepatic glycogen, plasma corticosterone and insulin in rats. J. Nutrit., 103, 866-874.

PERET J., CHANEZ M., COTA J., MACAIRE I., 1975. Effects of quantity and quality of dietary protein and variation in certain enzyme activities on glucose metabolism in the rat. J. Nutrit., 105, 15251534.

PERETIANU J., 1968. Teneur en corticostérone du sang et des surrénales chez le rat nourri avec un régime contenant un excès en méthionine. Arch. Sci. physiol., 22, 441-447.

ROGERS Q. R., LEUNG P. M. B., 1973. The influence of amino acids on the neuro regulation of food intake. Fed. Proc., 32, 1709-1719.

ROSEN F., ROBERTS N. R., NICHOL C. A., 1959. Glucocorticoids and transaminase activity. I. Increased activity of glutamic-pyruvic transaminase in four conditions associated with gluconeogenesis. J. biol. Chem., 234, 476-480.

ROSEN F., HARDING H. R., MILLHOLLAND R. J., NICHOL C. A., 1963. Glucocorticoids and transaminase activity. VI. Comparison of the adaptive increases of alanine and tyrosine $\alpha$ ketoglutarate transaminase. J. biol. Chem., 238, 3725-3729.

SCHIMKE R. T., 1970. Regulation of protein degradation in mammalian tissues, 178-228. In MUNRO H. N., Mammalian protein mefabolism, vol. 4. Acad. Press, New York, London.

SCHIMKE R. T., 1975. On the properties and mechanisms of protein turnover, 173-186. In SCHIMKE R. T., KATUNUMA N., Intracellular protein furnover, Acad. Press, New York, San Francisco.

SEGAL H. L., MATSUZAWA T., 1960. L-alanine amino transferase (rat liver), 155-159. In TABOR H., TABOR C. W., Methods in enzymology, vol. XVIla. Acad. Press, New York, London, 159.

SHRAGO E., LARDY H. A., NORDLIE R. C., FOSTER D. O., 1963. Metabolic and hormonal control of phosphoenolpyruvate carboxykinase and malic enzyme in rat liver., J. biol. Chem., 238, 31883192.

SNEDECOR G. W., COCHRAN W. G., 1967. Statistical methods. Ed. 6. lowa State Univ. Press, Ames, lowa.

SUDA M., NAKAWA H., 1971. Serine dehydratase (rat liver), 346-351. In TABOR H., TABOR C. W., Methods in Enzymol, vol. XVII (part B). Acad. Press, New York, London, San Francisco.

SZEPESI B., FREEDLAND R. A., 1967. Alterations in the activities of several rat liver enzymes at variations tissues after initiation of a high protein regimen. J. Nutrit., 93, 301-306.

SZEPESI B., FREEDLAND R. A., 1968o. Time-course of changes in rat liver enzyme activities after initiation of a high protein regimen. J. Nutrit, 94, 463-468.

SZEPESI B., FREEDLAND R. A., 1968b. Dietary regulation of pyruvate kinase synthesis in rat liver. J. Nutrit., 95, 591-602.

SZEPESI B., FREEDLAND R. A., 1969. A possible method for estimating hormone effects on enzyme synthesis. Arch. Biochem. Biophys., 133, 60-69.

TREADOW B. R., KHAIRALLAH E. A., 1972. Regulation of phosphoenolpyruvate carboxykinase during starvation and glucose repression. Nature (London). New Biol., 239, 131-133.

UNGER R. H., LEFEBVRE P., 1972. Glucagon physiology, 213-244. In LEFEBVRE P. J., UNGER R. H., Glucagon, molecular physiology, clinical and therapeutic implications. Pergamon Press, New York.

WEBER G., 1969. Regulation of pyruvate kinase. Adv. Enzyme Regul., 7, 15-40.

WEBER G., ALLARDE C., DE LAMIRANDE G., CANTERO A., 1955. Increased liver glucose-6phosphatase activity after cortisone administration. Biochim. biophys. Acta., 16, 618-624.

WEBER G., LEA M. A., HIRD CONVERY H. J., STAMM N. B., 1967. Regulation of gluconeogenesis and glycolysis : studies of mechanisms controlling enzyme activity. Adv. Enzyme Regul., 5, 257298.

WEBER G., SRIVASTAVA S. K., SINGHAL R. L., 1965a. Role of enzymes in homeostasis. VII. Early effects of cortical steroid hormones on hepatic gluconeogenic enzymes, ribonucleic acid metabolism and amino acid level. J. biol. Chem., 240, 750-755.

WEBER G., STAMM N. B., FISHER E. A., 1965b. Insulin : inducer of pyruvate kinase. Science, 149, 65-67.

WICKS W. D., KENNEY F. T., LEE K. L., 1969. Induction of hepatic enzyme. Synthesis in vivo by adenosine 3', 5'-monophosphate. J. biol. Chem., 244, 6008-6013.

WICKS W. D., LEWIS W., MC KIBBIN J. B., 1972. Induction of phosphoenolpyruvate carboxykinase by $\mathrm{N}^{6}-0^{2 \prime}$-dibutyryl cyclic AMP in rat liver. Biochim. biophys. Acta., 264, 171-185. 